\title{
AN EXPLORATORY EXAMINATION OF THE IMPACT OF VACATION POLICY Structure on SATISFACTION, Productivity, AND PROFITABILITY
}

\author{
Ashley Hurrell and John Keiser
}

The BRC Academy Journal of Business 10, no. 1 (2020): 33-63.

http://dx.doi.org/10.15239/j.brcacadjb.2020.10.02.ja02

\section{WEB APPENDIX}

https://dx.doi.org/10.15239/j.brcacadjb.2020.10.01.wa02 


\section{Appendix A: Top 100 Companies and their rankings in the 2017 Fortune 500 list}

\begin{tabular}{|c|c|c|}
\hline Walmart & 36 & Procter \& Gamble \\
\hline Berkshire Hathaway & 37 & Valero Energy \\
\hline Apple & 38 & Target \\
\hline Exxon Mobil & 39 & Freddie Mac \\
\hline McKesson & 40 & Lowe's \\
\hline UnitedHealth Group & 41 & Dell Technologies \\
\hline CVS Health & 42 & MetLife \\
\hline General Motors & 43 & Aetna \\
\hline AT\&T & 44 & PepsiCo \\
\hline Ford Motor & 45 & Archer Daniels Midland \\
\hline AmerisourceBergen & 46 & UPS \\
\hline Amazon.com & 47 & Intel \\
\hline General Electric & 48 & Prudential Financial \\
\hline Verizon & 49 & Albertsons Cos. \\
\hline Cardinal Health & 50 & United Technologies \\
\hline Costco & 51 & Marathon Petroleum \\
\hline Walgreens Boots Alliance & 52 & Disney \\
\hline Kroger & 53 & Humana \\
\hline Chevron & 54 & Pfizer \\
\hline Fannie Mae & 55 & AIG \\
\hline J.P. Morgan Chase & 56 & Lockheed Martin \\
\hline Express Scripts Holding & 57 & Sysco \\
\hline Home Depot & 58 & FedEx \\
\hline Boeing & 59 & Hewlett Packard Enterprise \\
\hline Wells Fargo & 60 & Cisco Systems \\
\hline Bank of America Corp. & 61 & HP \\
\hline Alphabet & 62 & Dow Chemical \\
\hline Microsoft & 63 & HCA Holdings \\
\hline Anthem & 64 & Coca-Cola \\
\hline Citigroup & 65 & New York Life Insurance \\
\hline Comcast & 66 & Centene \\
\hline IBM & 67 & American Airlines Group \\
\hline State Farm Insurance Cos. & 68 & Nationwide \\
\hline Phillips 66 & 69 & Merck \\
\hline Johnson \& Johnson & 70 & Cigna \\
\hline
\end{tabular}

$\begin{array}{ll}71 & \text { Delta Air Lines } \\ 72 & \text { Best Buy } \\ 73 & \text { Honeywell International } \\ 74 & \text { Caterpillar } \\ 75 & \text { Liberty Mutual Insurance } \\ & \text { Group } \\ 76 & \text { Morgan Stanley } \\ 77 & \text { Massachusetts Mutual } \\ & \text { Life Insurance } \\ 78 & \text { Goldman Sachs Group } \\ 79 & \text { Energy Transfer Equity } \\ 80 & \text { TIAA } \\ 81 & \text { Oracle } \\ 82 & \text { Tyson Foods } \\ 83 & \text { United Continental } \\ & \text { Holdings } \\ 84 & \text { Allstate } \\ 85 & \text { Publix Super Markets } \\ 86 & \text { American Express } \\ 87 & \text { TJX } \\ 88 & \text { Nike } \\ 89 & \text { Exelon } \\ 90 & \text { General Dynamics } \\ 91 & \text { Rite Aid } \\ 92 & \text { Gilead Sciences } \\ 93 & \text { CHS } \\ 94 & \text { 3M } \\ 95 & \text { Time Warner } \\ 96 & \text { Charter Communications } \\ 97 & \text { Northwestern Mutual } \\ 98 & \text { Facebook } \\ 99 & \text { Travelers Cos. } \\ 100 & \text { Capital One Financial } \\ & \end{array}$




\section{Appendix B: Statistical Analyses}

Table 1

Summary results of t-tests and Descriptive Statistics for Policy Satisfaction, Employee Productivity, and Organizational Profitability by policy structure

\begin{tabular}{|c|c|c|c|c|c|c|c|c|c|}
\hline \multirow[t]{3}{*}{ Outcome } & \multicolumn{6}{|c|}{ Group } & \multirow[b]{3}{*}{$\mathrm{t}$} & \multirow[b]{3}{*}{$\mathrm{p}$} & \multirow[b]{3}{*}{$\mathrm{df}$} \\
\hline & \multicolumn{3}{|c|}{ Traditional } & \multicolumn{3}{|c|}{ PTO Bank } & & & \\
\hline & $\mathrm{M}$ & SD & $\mathrm{n}$ & $\mathrm{M}$ & SD & $\mathrm{n}$ & & & \\
\hline Policy Satisfaction & 3.8644 & 0.4310 & 59 & 3.8543 & 0.4967 & 35 & 0.104 & 0.917 & 92 \\
\hline Employee Productivity & 147,260 & 338,568 & 59 & 49,704 & 45,972 & 35 & 2.180 & 0.033 & 92 \\
\hline Organizational Profitability & 0.1043 & 0.0901 & 59 & 0.0594 & 0.0610 & 35 & 2.875 & 0.005 & 92 \\
\hline
\end{tabular}


Table 2

Pearson Correlation Matrix of Policy Satisfaction, Employee Productivity, and Organizational Profitability

\begin{tabular}{|c|c|c|c|c|}
\hline & & Policy & Employee & Organizational \\
\hline & & Satisfaction & Productivity & Profitability \\
\hline Policy & Pearson Correlation & 1 & 0.203 & 0.265 \\
\hline \multirow[t]{2}{*}{ Satisfaction } & Sig. (2-tailed) & & 0.049 & 0.009 \\
\hline & $\mathrm{N}$ & 95 & 95 & 95 \\
\hline Employee & Pearson Correlation & 0.203 & 1 & 0.465 \\
\hline \multirow[t]{2}{*}{ Productivity } & Sig. (2-tailed) & 0.049 & & 0.000 \\
\hline & $\mathrm{N}$ & 95 & 95 & 95 \\
\hline Organizational & Pearson Correlation & 0.265 & 0.465 & 1 \\
\hline \multirow[t]{2}{*}{ Profitability } & Sig. (2-tailed) & 0.009 & 0.000 & \\
\hline & $\mathrm{N}$ & 95 & 95 & 95 \\
\hline
\end{tabular}

